Research Article

\title{
The Use of Oral Analgesics and Pain Self-Efficacy Are Independent Predictors of the Quality of Life of Individuals with Rheumatoid Arthritis
}

\author{
Akira Hashimoto, Motoki Sonohata $(D$, and Masaaki Mawatari \\ Department of Orthopaedic Surgery, Faculty of Medicine, Saga University, Nabeshima 5-1-1, Saga 849-8501, Japan \\ Correspondence should be addressed to Motoki Sonohata; epc9719@yahoo.co.jp
}

Received 17 March 2020; Accepted 1 July 2020; Published 24 July 2020

Academic Editor: Shinya Kasai

Copyright ( $\odot 2020$ Akira Hashimoto et al. This is an open access article distributed under the Creative Commons Attribution License, which permits unrestricted use, distribution, and reproduction in any medium, provided the original work is properly cited.

\begin{abstract}
Objectives. This study investigated the relationship between quality of life (QOL) and several factors, including pain assessments, in patients with rheumatoid arthritis (RA). Methods. This cross-sectional, single-center study enrolled 85 patients with RA. The variables investigated included demographic characteristics, the 28-joint disease activity score with C-reactive protein (DAS28CRP), painDETECT questionnaire (PDQ), pain self-efficacy questionnaire (PSEQ), and pain catastrophizing scale (PCS). QOL was measured using the Japanese validated version of the European Quality of Life questionnaire with five dimensions and five levels (EQ-5D-5L). Results. The use of oral steroids and oral analgesics was significantly associated with low EQ-5D-5L scores $(P<0.05)$. EQ-5D-5L score had a significant positive association with PSEQ $(r=0.414)$ and significant negative association with age, disease duration, DAS28-CRP, PDQ, and PCS $(r=-0.217,-0.343,-0.217,-0.277$, and -0.384 , respectively). Multiple regression analysis showed that the use of oral analgesics and PSEQ were independent predictors of EQ-5D-5L score $(\beta=-0.248$, $P<0.05$ and $\beta=0.233, P<0.05)$. Conclusions. The use of oral analgesics by RA patients may influence their QOL, which, in turn, may affect their feelings of self-efficacy. Various pain management strategies, including surgical treatment, may be explored for the treatment of RA. Furthermore, the PSEQ may be a prominent part of the patient's overall assessment.
\end{abstract}

\section{Introduction}

Rheumatoid arthritis (RA) is a chronic, progressive, systemic inflammatory autoimmune disease that causes joint deformity, pain, and functional disability $[1,2]$. RA affects approximately $0.5-1 \%$ of the population globally [3]. The combination of methotrexate and biological disease-modifying antirheumatic drugs (bDMARDs) has contributed to an increase in the number of patients who achieve clinical remission [4]. Disease activity in RA is often assessed using the disease activity score in 28 joints (DAS28) [5], which can further be combined with the C-reactive protein level (DAS28-CRP). However, $12.5 \%$ of patients with RA continue to report clinically significant pain despite achieving remission according to the DAS28-CRP score for more than 1 year [6]. A previous report examining patients with RA found that even those with low disease activity may have neuropathic pain [7]. Pain is usually associated with poor quality of life (QOL) in patients with RA [8].
Self-efficacy beliefs and pain catastrophizing also affect QOL in patients with RA $[9,10]$. Satisfactory QOL is an important component of remission; complete remission of RA is defined as the achievement of clinical, structural, and functional remission [11]. Therefore, the main goal of RA treatment is the improvement of the patient's QOL [12]. However, the overall benefit of intensive treatment strategies in RA remains uncertain [13].

One of the common health-related QOL (HRQOL) measurement tools is the European Quality of Life questionnaire with five dimensions and five different response options (EQ-5D-5L) [14-16]. Several reports have described the use of the EQ-5D-5L for assessing the QOL of patients with RA [17-19]. However, those reports included a limited number of factors [17-19], and none have investigated the relationship between the current study variables, such as pain self-efficacy or pain catastrophizing, and QOL using the EQ-5D-5L.

In this study, we aimed to investigate the variables that influence the QOL of patients with RA, using the EQ-5D-5L. 


\section{Materials and Methods}

2.1. Patients and Methods. This single-center study utilized a cross-sectional design and was conducted at our institution in October 2016. The study protocol adhered to the ethical guidelines of the 1975 Declaration of Helsinki; the study design was approved by the appropriate ethics institutional review board (No. 2016-02-02), and all study participants provided informed consent.

A total of 87 patients with RA were recruited for this study. Two patients were excluded from the analyses due to incomplete data, and 85 patients were included in the final analyses. Aside from a lack of data, there were no other exclusion criteria. All patients had a confirmed diagnosis of RA according to the 2010 American College of Rheumatology/European League Against Rheumatism [20]. The study variables included age, sex, body mass index (BMI), disease duration, presence and number of RA-related operations, use of MTX, use of bDMARDs, use of oral steroids, use and type of oral analgesics, use of synthetic DMARDs (sDMARDs) in patients with oral analgesics, serum matrix metalloprotease-3 (MMP-3) levels, EQ-5D-5L, DAS28-CRP, painDETECT questionnaire (PDQ), pain self-efficacy questionnaire (PSEQ), and pain catastrophizing scale (PCS).

\subsection{Measurement and Assessment Tools}

2.2.1. EQ-5D-5L. The EQ-5D-5L is a common HRQOL measurement tool. The EQ-5D-5L measures HRQOL using five items: mobility, self-care, usual activities, pain/discomfort, and anxiety/depression [15]. Each item has five different response options: no problems, slight problems, moderate problems, severe problems, and unable to/extreme problems; the combination of responses yields 3125 unique health statuses [15]. A Japanese version of the EQ-5D-5L has been developed [16]. The total scores range from 0 to 1 [16], and a higher score indicates a better QOL.

2.2.2. DAS28-CRP. The DAS28-CRP is an assessment of disease activity in RA. DAS28-CRP scores are calculated by assessing three components: (1) the number of swollen and tender joints, out of 28 , as determined by a trained rheumatologist; (2) the patient Visual Analog Scale for general disease activity; and (3) CRP levels [5, 21]. RA activity is categorized according to the DAS28-CRP score as remission $(<2.3)$, low (2.4-2.7), moderate (2.8-4.1), or high (>4.1) [22]. In this study, DAS28-CRP was evaluated as a continuous variable, without dividing it into categories.

2.2.3. PDQ. The PDQ is an assessment for measuring noninflammatory, neuropathic, or sensitization elements of pain [23]. The PDQ contains seven items evaluating pain qualities, one evaluating the course pattern of pain, and one evaluating pain radiation [23]. The questionnaire contains three 0-10 numerical rating scales for current, worst, and average pain severity. An overall score is generated, which summarizes everything but the pain intensity numerical rating scales, and this overall score ranges between -1 and 38. An overall score $>18$ indicates likely neuropathic pain, 13-18 possible neuropathic pain, and $<13$ unlikely neuropathic pain [23]. In this study, PDQ was evaluated as a continuous variable.

2.2.4. PSEQ. The PSEQ is an assessment of the patient's confidence in performing activities despite pain [24]. It is a 10-item questionnaire; each item is rated on a 7-point numerical rating scale. A higher score indicates higher selfefficacy or more confidence in managing chronic conditions. The total scores range from 0 to 60 . The Cronbach's $\alpha$ coefficient for the Japanese version of the PSEQ is 0.94 [25].

2.2.5. PCS. The PCS is an assessment of the degree of catastrophic thinking regarding pain. It comprises 13 items, each of which is rated on a 5-point numerical rating scale. The PCS has three subscales: rumination, magnification, and helplessness [26]. Higher scores indicate greater levels of catastrophizing. The total scores range from 0 to 51 . The Cronbach's $\alpha$ coefficients for the Japanese version of the PCS are 0.80 for the rumination subscale, 0.65 for the magnification subscale, 0.81 for the helplessness subscale, and 0.89 for the total score [27].

2.3. Statistical Analysis. All numerical data were expressed as the mean \pm standard deviation. All statistical analyses were performed using SPSS version 23 for Windows (International Business Machines Corporation (IBM), NY, USA). The Kolmogorov-Smirnov test was conducted to evaluate the distribution normality of the continuous variables. Independent $t$-tests were used to compare the EQ-5D-5L scores by sex, history of any RA-related operations, use of MTX, use of bDMARDs, use of oral steroids, and use of oral analgesics. Independent $t$-tests were also used to compare the use of oral analgesics with the individual items of the PSEQ. Pearson correlation coefficients were obtained to assess the correlations between the EQ-5D-5L and age, BMI, disease duration, number of RA-related operations, MMP-3 levels, DAS28CRP, PDQ, PSEQ, and PCS; between DAS28-CRP, PDQ, PSEQ, and PCS; and between EQ-5D-5L and the score of each of the items of the PSEQ. Tukey's test was used to compare the scores among the 10 items of the PSEQ.

We performed a multivariate analysis to identify the variables that independently predict the EQ-5D-5L scores using multiple linear regression $[28,29]$. The variables included in the multivariate analysis were those with a $P$ value $<0.05$ in the univariate analyses $[28,29]$. The level of significance was set at $P<0.05$.

\section{Results}

The demographic characteristics of the patients are shown in Table 1. The type of oral analgesics used and the percentage of patients who also use sDMARDs in addition to oral analgesics are shown in Table 2 . The percentage of patients who used oral analgesics was $29.4 \%$. The overall percentage of patients who use sDMARDs out of all patients who use oral analgesics was $92.0 \%$. The percentage of patients who 
Table 1: Participant characteristics.

Number of patients

Sex (males, females; $n(\%)$ )

Age (years; mean $\pm \mathrm{SD}$, range)

$\mathrm{BMI}\left(\mathrm{kg} / \mathrm{m}^{2}\right.$; mean $\pm \mathrm{SD}$, range $)$

Disease duration (months; mean $\pm \mathrm{SD}$, range)

History of any RA-related operation, $n$ (\%)

Number of RA-related operations (mean $\pm S D$, range)

Use of MTX, $n$ (\%)

Use of bDMARDs, $n(\%)$

Use of oral steroids, $n(\%)$

Use of oral analgesics, $n(\%)$

MMP-3 levels (ng/mL; mean $\pm \mathrm{SD}$, range)

EQ-5D-5L (mean \pm SD, range)

$\mathrm{DAS} 28-\mathrm{CRP}$ (mean $\pm \mathrm{SD}$, range)

$\mathrm{PDQ}$ (mean $\pm \mathrm{SD}$, range)

PSEQ (mean $\pm \mathrm{SD}$, range)

PCS (mean $\pm S D$, range)
85

11 (14.9), $74(85.1)$

$63.0 \pm 12.3,17-85$

$22.3 \pm 4.0,14.7-38.5$

$140.1 \pm 116.5,7-582$

$40(46.0)$

$0.9 \pm 1.3,0-6$

$55(63.2)$

$46(52.9)$

$9(10.3)$

$25(29.4)$

$63.4 \pm 45.1,12.8-311.9$

$0.8096 \pm 0.1570,0.1722-0.9384$

$1.3 \pm 0.9,1.0-5.3$

$5.3 \pm 4.5,0-18$

$42.8 \pm 13.0,9-60$

$15.5 \pm 11.2,0-40$

SD: standard deviation, BMI: body mass index, RA: rheumatoid arthritis, MTX: methotrexate, bDMARDs: biological disease-modifying antirheumatic drugs, MMP-3: serum matrix metalloprotease-3, EQ-5D-5L: the European Quality of Life questionnaire, five dimensions, five levels, DAS28-CRP: disease activity score based on the 28-joint assessment-C-reactive protein, PDQ: painDETECT questionnaire, PSEQ: pain self-efficacy questionnaire, PCS: pain catastrophizing scale.

TABLE 2: Type of oral analgesics and use of sDMARDs in patients using oral analgesics.

\begin{tabular}{lcc}
\hline & $\begin{array}{c}\text { Number of patients who use oral } \\
\text { analgesics }\end{array}$ & $\begin{array}{c}\text { Number of patients who also use sDMARDs among patients using oral } \\
\text { analgesics }\end{array}$ \\
\hline $\begin{array}{l}N(\%) \\
\text { Type of oral analgesics }\end{array}$ & $25(29.4)^{*}$ & $23(92)^{\dagger}$ \\
\hline $\begin{array}{l}\text { NSAIDs, } n(\%) \\
\begin{array}{l}\text { Acetaminophen, } n \\
(\%)\end{array}\end{array}$ & $3(12)$ & $3(100)$ \\
Pregabalin, $n(\%)$ & $3(12)$ & $2(67)$ \\
\hline
\end{tabular}

sDMARDs: synthetic disease-modifying antirheumatic drugs, NSAIDs: non-steroidal anti-inflammatory drug. *Percentages in this column represent the percentage of the whole patient sample $(N=85)$; †percentages in this column represent the percentage of the patients who take oral analgesics or each specific type of oral analgesic.

use sDMARDs out of all patients who use nonsteroidal antiinflammatory drugs (NSAIDs) was $94.7 \%$.

The results of the independent $t$-tests between the EQ-5D5L and the studied variables are shown in Table 3 and Figure 1. The use of oral steroids and oral analgesics was significantly associated with low EQ-5D-5L scores (both $P<0.05$ ).

Pearson correlation coefficients between the EQ-5D-5L and studied variables are shown in Figure 2. The correlations were significant for age, disease duration, DAS28-CRP, PDQ, PSEQ, and PCS $(r=-0.217,-0.343,-0.217,-0.277$, 0.414 , and -0.384 , respectively). Results of the multiple regression analysis between the EQ-5D-5L and study variables are shown in Table 4. Among these, the use of oral analgesics and PSEQ scores were independent predictors $(\beta=-0.248, P<0.05$ and $\beta=0.233, P<0.05)$.

Pearson correlation coefficients among the DAS28-CRP, PDQ, PSEQ, and PCS are shown in Figure 3. PSEQ had a significant positive correlation only with PCS $(r=-0.345$, $P<0.05)$. There was no significant correlation between PSEQ and DAS28-CRP.

There were significant positive correlations between the EQ-5D-5L and all items of the PSEQ $(P<0.05)$ (Table 5). The score on question 7 of the PSEQ ("I can cope with my pain without medication.") was significantly lower than that on all other questions $(P<0.05)$ (Table 5$)$. For the score on question 7 of the PSEQ, patients who used oral analgesics $(2.48 \pm 1.73$, range; $0-6)$ had significantly lower scores than those who did not $(3.45 \pm 1.67$, range; $0-6)(P<0.018)$.

\section{Discussion}

Multiple previous studies have reported clinical and imaging remission in patients with $\mathrm{RA}$, but few studies have focused on functional remission $[4,30]$. In the present study, we investigated important factors associated with QOL in patients with RA, and our findings suggest that the disease duration, PDQ, PSEQ, PCS, use of oral steroids, and use of oral analgesics play important roles in achieving functional remission. Among these factors, the use of oral analgesics and PSEQ scores had particularly strong associations with QOL in patients with RA.

There are numerous tools to measure RA activity available for use [31]. The DAS28-CRP is widely used to evaluate RA activity and is a useful tool for assessing patients with RA [32]. The DAS28-CRP has several advantages: it is simple and quick, is useful for evaluating disease activity, 
TABLE 3: Independent $t$-tests between EQ-5D-5L and studied variables.

\begin{tabular}{|c|c|c|c|c|c|c|}
\hline \multirow{2}{*}{ Parameters } & \multirow{2}{*}{ Number of patients $n(\%)$} & \multicolumn{4}{|c|}{ EQ-5D-5L } & \multirow{2}{*}{$P$ value } \\
\hline & & Mean & Median & Standard deviation & Range & \\
\hline \multicolumn{7}{|l|}{ Sex } \\
\hline Female & $74(87.1)$ & 0.8008 & 0.8406 & 0.1595 & $0.1722-0.9384$ & 0.183 \\
\hline Male & $11(12.9)$ & 0.8686 & 0.9384 & 0.1303 & $0.5960-0.9384$ & \\
\hline \multicolumn{7}{|c|}{$R A$-related operations } \\
\hline Absence & $45(52.9)$ & 0.8403 & 0.8973 & 0.1205 & $0.4168-0.9384$ & 0.055 \\
\hline Presence & $40(47.1)$ & 0.7750 & 0.8365 & 0.1854 & $0.1722-0.9384$ & \\
\hline \multicolumn{7}{|l|}{ Use of MTX } \\
\hline Absence & $30(35.3)$ & 0.7782 & 0.8324 & 0.1666 & $0.2466-0.9384$ & 0.175 \\
\hline Presence & $55(64.7)$ & 0.8267 & 0.8973 & 0.1503 & $0.1722-0.9384$ & \\
\hline \multicolumn{7}{|c|}{ Use of bDMARDs } \\
\hline Absence & $46(54.1)$ & 0.8305 & 0.8406 & 0.1267 & $0.4168-0.9384$ & 0.261 \\
\hline Presence & $39(45.9)$ & 0.7919 & 0.8555 & 0.1782 & $0.1722-0.9384$ & \\
\hline \multicolumn{7}{|c|}{ Use of oral steroids } \\
\hline Absence & $76(89.4)$ & 0.8299 & 0.8893 & 0.1358 & $0.1722-0.9384$ & 0.032 \\
\hline Presence & $9(10.6)$ & 0.6383 & 0.7026 & 0.2213 & $0.2466-0.9384$ & \\
\hline \multicolumn{7}{|c|}{ Use of oral analgesics } \\
\hline Absence & $60(70.6)$ & 0.8456 & 0.8973 & 0.1235 & $0.4168-0.9384$ & 0.001 \\
\hline Presence & $25(29.4)$ & 0.7233 & 0.7752 & 0.1944 & $0.1722-0.9384$ & \\
\hline
\end{tabular}

EQ-5D-5L: the European Quality of Life questionnaire, five dimensions, five levels, RA: rheumatoid arthritis, MTX: methotrexate, bDMARDs: biological disease-modifying antirheumatic drugs.

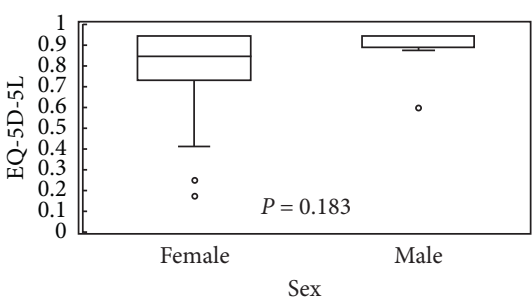

(a)

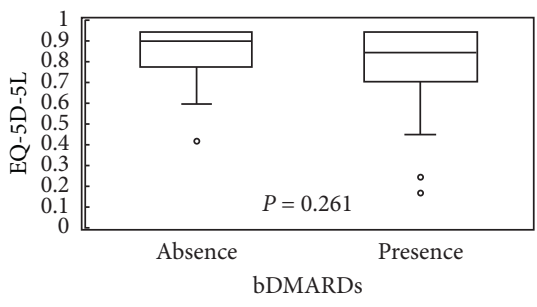

(d)

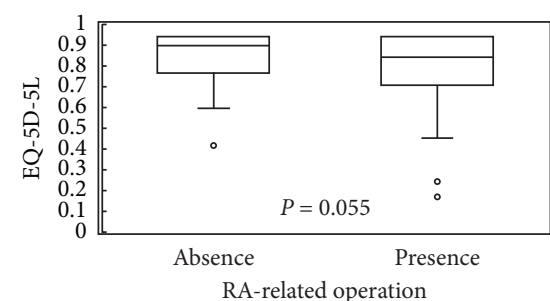

(b)

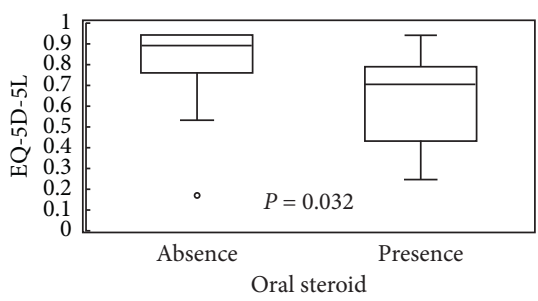

(e)

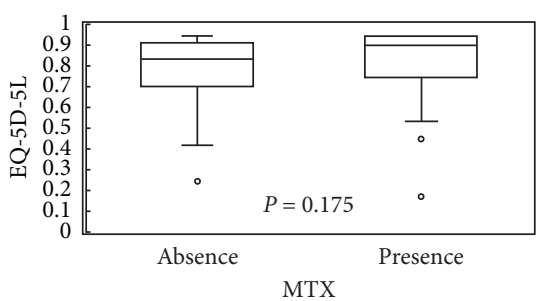

(c)

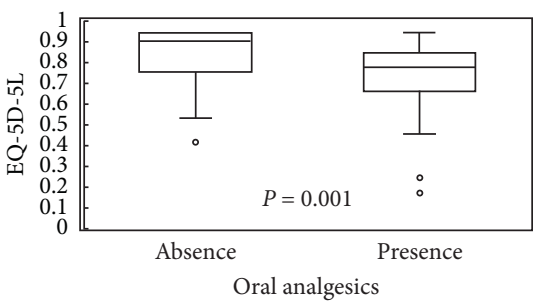

(f)

FIgURE 1: Box-and-whisker plots showing differences in EQ-5D-5L for the dichotomous study variables. (a) Sex, (b) history of any RArelated operations, (c) use of MTX, (d) use of bDMARDs, (e) use of oral steroids, and (f) use of oral analgesics. Data are presented as the mean \pm standard deviation. EQ-5D-5L: the European Quality of Life questionnaire, five dimensions, five levels; RA: rheumatoid arthritis; MTX: methotrexate; bDMARDs: biological disease-modifying antirheumatic drugs.

and correlates with radiological progression [33, 34]. However, the disadvantages of the DAS28-CRP are its exclusion of the ankle and foot joints and less stringent remission criteria compared with the Simplified Disease Activity Index and Clinical Disease Activity Index [35, 36]. In addition, up to $40 \%$ of patients with RA demonstrated a progressive erosive disease detected by magnetic resonance imaging (MRI) despite DAS28-CRP improvement or EULAR remission [37]. Thus, other assessments should be included in the evaluation of patients with RA, such as the
PSEQ and EQ-5D-5L, to provide a more complete description of the patient's overall recovery.

Pain in patients with RA is traditionally thought to be nociceptive pain of inflammatory origin [38]. However, $12.5 \%$ of patients with RA who achieve DAS28-CRP remission have clinically significant pain, and this pain may be neuropathic $[6,39]$. Moreover, patients with RA who have medium and high PDQ scores have worse indicators of anxiety, depression, disability, HRQOL, pain, and fatigue [40]. Therefore, only utilizing the DAS28-CRP for the assessment and treatment of 


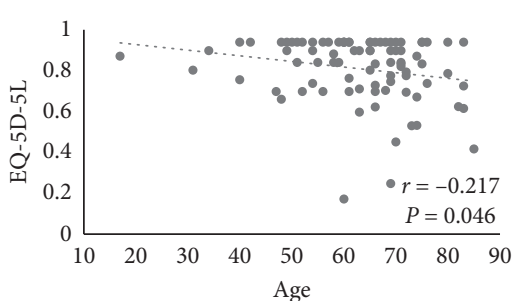

(a)

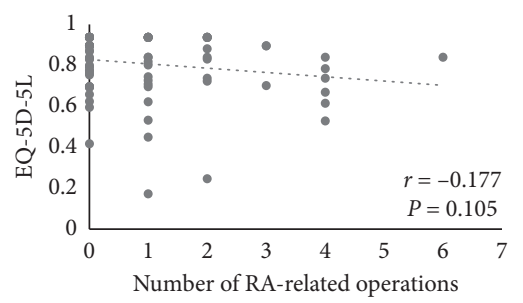

(d)

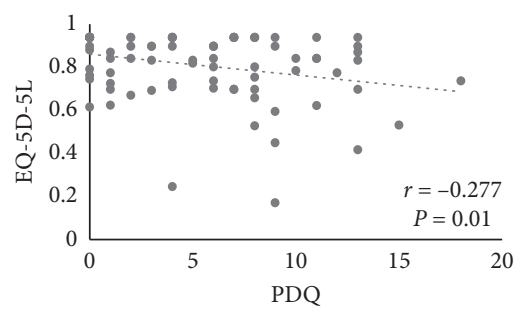

(g)

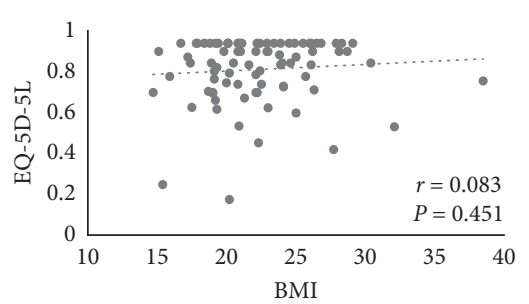

(b)

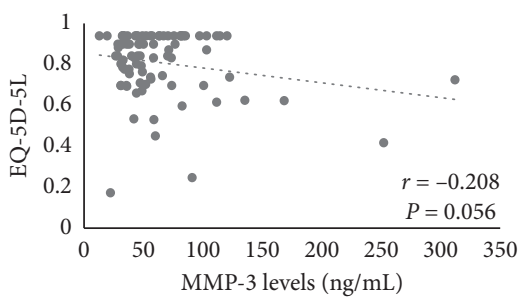

(e)

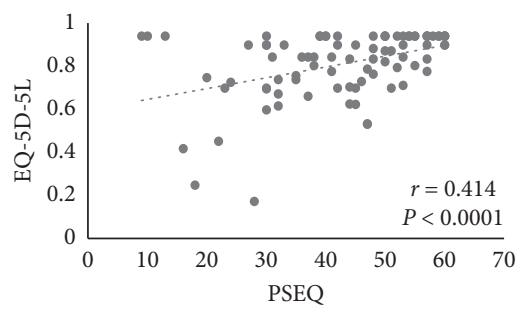

(h)

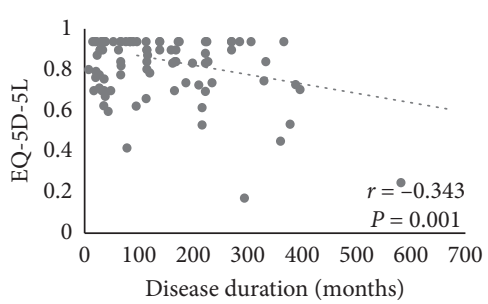

(c)

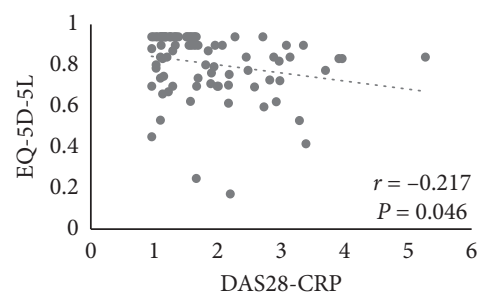

(f)

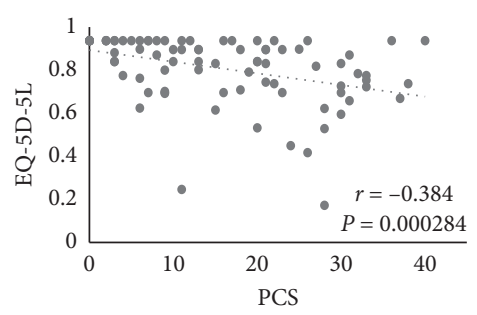

(i)

Figure 2: Correlations between the EQ-5D-5L and continuous study variables. (a) Age, (b) BMI, (c) disease duration, (d) number of RArelated operations, (e) MMP-3 levels, (f) DAS28-CRP, (g) PDQ, (h) PSEQ, and (i) PCS. EQ-5D-5L: the European Quality of Life questionnaire, five dimensions, five levels; BMI: body mass index; RA: rheumatoid arthritis; MTX: methotrexate; MMP-3: serum matrix metalloprotease-3; DAS28-CRP: the disease activity score based on the 28-joint assessment-C-reactive protein; PDQ: painDETECT questionnaire; PSEQ: pain self-efficacy questionnaire; PCS: pain catastrophizing scale.

TABLE 4: Summary of the multiple regression analysis between the EQ-5D-5L and variables of interest.

\begin{tabular}{lcccc}
\hline & $\mathrm{B}$ & SE B & $\mathrm{B}$ & $P$ value \\
\hline Age & -0.001 & 0.001 & -0.074 & 0.414 \\
Disease duration & 0 & 0 & -0.159 & 0.122 \\
Use of oral steroids & -0.068 & 0.055 & 0.134 & 0.220 \\
Use of oral analgesics & -0.085 & 0.031 & $-0.248^{*}$ & 0.008 \\
DAS28-CRP & -0.006 & 0.017 & -0.033 & 0.730 \\
PDQ & -0.005 & 0.003 & -0.139 & 0.162 \\
PSEQ & 0.003 & 0.001 & $0.233^{*}$ & 0.019 \\
PCS & -0.003 & 0.001 & -0.207 & 0.056 \\
$R^{2}=0.371$ & & & &
\end{tabular}

EQ-5D-5L: the European Quality of Life questionnaire, five dimensions, five levels; MTX: methotrexate; DAS28-CRP: the disease activity score based on the 28-joint assessment-C-reactive protein; PDQ: painDETECT questionnaire; PSEQ: pain self-efficacy questionnaire; PCS: pain catastrophizing scale. ${ }^{*}$ Significant difference between the EQ-5D-5L and studied variable $(P<0.05)$.

RA disease activity and only treating nociceptive pain may fail to improve QOL. Indeed, the assessment and treatment of pain (both nociceptive and neuropathic) with the goal of clinical remission are necessary for improving QOL.

In this study, psychosocial factors such as pain catastrophizing (PC) and pain self-efficacy (PSE), represented by the PCS and PSEQ scores, respectively, influenced the patients' QOL. Bandura [41] defined self-efficacy as "people's judgments of their capabilities to organize and execute courses of action required to attain designated types of performances." PSE is patients' confidence that they can achieve their goals despite their pain [42]. PSE is associated with pain severity, pain intensity, negative psychological factors, and disability [9, 43-45]. In addition, high PSE is associated with a better QOL [9], and PSE is an important factor to consider for patients with RA [46].

PC is defined as "a set of exaggerated and negative cognitive and emotional schema brought to bear during actual or anticipated painful stimulation" [47]. PC is associated with pain intensity, depression, and anxiety [48]. One study found that one-fourth of patients with RA have high PCS, despite biotherapy [49]. Thus, PSE and PC are associated with pain and QOL, and pain management that can both increase PSE and reduce PC may ultimately improve QOL. One such strategy is acceptance and commitment therapy (ACT), which is a form of cognitive behavioural therapy [50]. ACT reduces pain intensity and increases selfefficacy [51, 52]. ACT also reduces PC and depression $[50,53]$. Therefore, ACT would be useful for improving QOL in patients with RA. 


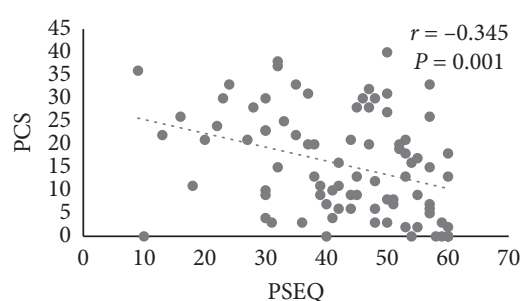

(a)

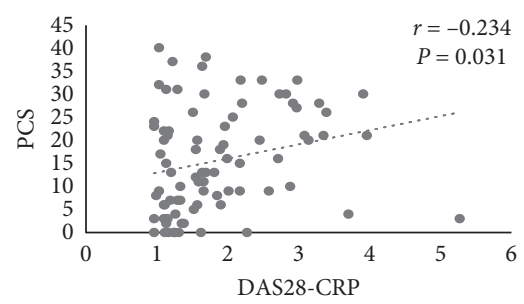

(d)

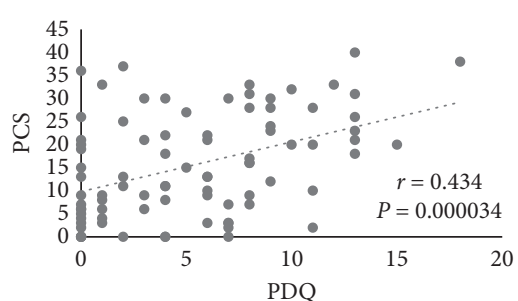

(b)

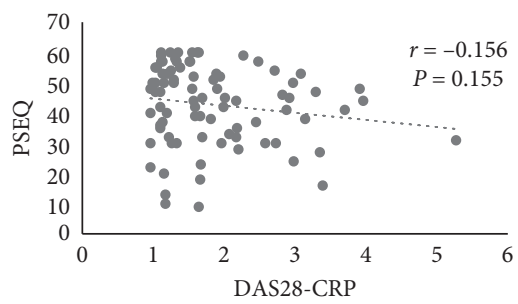

(e)

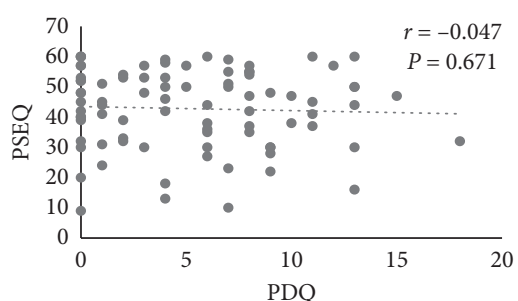

(c)

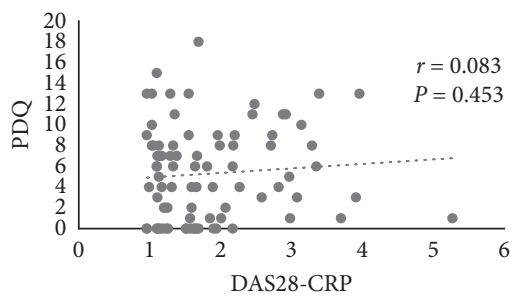

(f)

Figure 3: Correlations among the DAS28-CRP, PDQ, PSEQ, and PCS. DAS28-CRP: the disease activity score based on the 28-joint assessment-C-reactive protein; PDQ: painDETECT questionnaire; PSEQ: pain self-efficacy questionnaire; PCS: pain catastrophizing scale.

TABLE 5: Comparisons between individual items of the PSEQ.

\begin{tabular}{|c|c|c|c|c|c|c|}
\hline \multirow[b]{2}{*}{ Parameters } & \multicolumn{4}{|c|}{ Score } & \multirow[b]{2}{*}{$\begin{array}{c}P \text { value between Q7 and each item } \\
\text { (Tukey's test) }\end{array}$} & \multirow[b]{2}{*}{$\begin{array}{l}\text { Correlation between EQ-5D-5L and each item } \\
\text { (Pearson correlation) }\end{array}$} \\
\hline & Mean & Median & $\begin{array}{l}\text { Standard } \\
\text { deviation }\end{array}$ & Range & & \\
\hline Q1 & 4.3529 & 5.0 & 1.5408 & $0-6$ & $P<0.001$ & $r=0.348, P=0.001$ \\
\hline Q2 & 4.4471 & 5.0 & 1.5237 & $0-6$ & $P<0.001$ & $r=0.383, P<0.001$ \\
\hline Q3 & 4.7529 & 5.0 & 1.3085 & $0-6$ & $P<0.001$ & $r=0.228, P=0.036$ \\
\hline Q4 & 4.4235 & 5.0 & 1.4005 & $0-6$ & $P<0.001$ & $r=0.271, P=0.012$ \\
\hline Q5 & 4.2941 & 5.0 & 1.6462 & $0-6$ & $P<0.001$ & $r=0.437, P<0.001$ \\
\hline Q6 & 4.5529 & 5.0 & 1.4268 & $1-6$ & $P<0.001$ & $r=0.379, P<0.001$ \\
\hline Q7 & 3.1647 & 3.0 & 1.7379 & $0-6$ & $P=1$ & $r=0.337, P=0.002$ \\
\hline Q8 & 4.0706 & 4.0 & 1.6315 & $0-6$ & $P=0.044$ & $r=0.366, P=0.001$ \\
\hline Q9 & 4.4418 & 5.0 & 1.4662 & $0-6$ & $P<0.001$ & $r=0.404, P<0.001$ \\
\hline Q10 & 4.3176 & 5.0 & 1.4817 & $0-6$ & $P=0.001$ & $r=0.359, P=0.001$ \\
\hline
\end{tabular}

PSEQ: pain self-efficacy questionnaire; Q: question. EQ-5D-5L: the European Quality of Life questionnaire, five dimensions, five levels.

In the present study, the EQ-5D-5L score was correlated with DAS28-CRP, PDQ, PSEQ, and PCS scores. PSEQ was not correlated with DAS28-CRP. Moreover, the multiple regression analysis revealed that PSEQ was an independent predictor of QOL for patients with RA. This provides evidence that assessing only DAS28-CRP and treating RA disease activity without considering PSEQ are insufficient for improving QOL. PCS was also correlated with DAS28CRP, PDQ, and PSEQ. The correlation between PCS and PDQ was stronger than the correlation between PCS and DAS28-CRP or PSEQ. Therefore, the assessment and treatment of pain (both nociceptive and neuropathic) could reduce PCS and lead to an improvement in QOL.

In a previous report, a higher daily dose of oral steroids was associated with a low QOL [54]. In this study, the use of oral steroids was significantly associated with low EQ-5D-5L scores. Past reports have described the effectiveness of methotrexate with step-down oral steroids for the initial treatment of RA [55]. It may be better for a patient's QOL to avoid long-term administration of oral steroids if clinical remission could be achieved and maintained without use of oral steroids.

The use of oral analgesics was significantly associated with low EQ-5D-5L scores in this study. A previous study indicated that therapy with sDMARDs in combination with NSAIDs was associated with depression in patients with RA [56]. Depression is associated with PC and reduced QOL [48]. In this study, 19 of 25 patients who used oral analgesics also used NSAIDs, and 18 of those 19 patients were being treated with sDMARDs. Currently, therapy utilizing sDMARDs and bDMARDs represents the primary treatment for RA [57]. However, pain control with only NSAIDs may fail to achieve sufficient QOL in patients with RA. Therefore, other pain control measures without NSAIDs may be needed.

Concerning RA-related operations, there was no significant difference in QOL between patients who had and had not undergone one or more RA-related operations. Although the history of an RA-related operation appears to have no association with QOL, this also indicates that patients who have undergone an RA-related operation can 
achieve a QOL equivalent to those who did not require an operation.

The scores on question 7 of the PSEQ were significantly lower than the scores on the other items. Moreover, the scores on Question 7 of the PSEQ were significantly lower for patients who were taking oral analgesics than those for patients who were not. This could indicate a possible mechanism, such that the use of oral analgesics was associated with the patient's low feelings of self-efficacy, resulting in a lower QOL. Therefore, pain control with oral analgesics may provide less improvement in QOL, and rheumatologists should carefully consider the possibility of surgical intervention.

In this study, long disease duration and older age were risk factors for poor QOL, which is consistent with previous studies $[58,59]$. Long disease duration is an important factor that increases symptoms of depression in patients with RA [60], and depression negatively impacts QOL [61]. In addition, elderly individuals usually have a poorer QOL [62], and they are more likely to feel pain than younger patients [63]. Pain and QOL are closely related, as described above. However, disease duration and age are factors that cannot be controlled. Therefore, elderly patients with RA and those with long disease duration may require a distinct overall treatment model.

The main strength of this study was the number of new variables studied in relation to QOL, which was assessed using the EQ-5D-5L. This study also has some limitations. First, the study population was relatively small, with a low number of male patients. It was a single-center study; therefore, a multicenter study may be needed to examine a large, diverse group of patients in the future. Second, almost all of the patients in this study had a well-controlled DAS28-CRP (mean score: 1.3 \pm 0.9 ; range: 1.0-5.3). Therefore, it may not be a fully representative patient group. However, the majority of patients with RA achieve wellcontrolled RA disease activity due to the dramatic changes in RA treatment over the past 20 years [64]. Third, the patients' history of major depression was not investigated, and depression is expected to reduce QOL. Finally, this study obtained only cross-sectional data. Therefore, a prospective study may be needed in the future.

\section{Conclusions}

PSEQ scores and the use of oral analgesics are independent predictors of QOL in patients with RA. Pain control with only oral analgesics may lead to reduced QOL. Using only the DAS28-CRP for the assessment and treatment of RA disease activity may not adequately support the patient's QOL during RA treatment. Therefore, various pain management strategies, including surgical treatment, may be considered for the treatment of RA. Furthermore, the PSEQ may be included in the assessment of the efficacy of RA treatments.

\section{Data Availability}

The data used to support the findings of this study are available from the corresponding author upon request.

\section{Conflicts of Interest}

The authors declare that there are no conflicts of interest regarding the publication of this paper.

\section{References}

[1] D. L. Scott, F. Wolfe, and T. W. Huizinga, "Rheumatoid arthritis," The Lancet, vol. 376, no. 9746, pp. 1094-1108, 2010.

[2] A. C. Tang, H. Kim, B. Crawford, T. Ishii, and T. Treuer, "The use of patient reported outcome measures for rheumatoid arthritis in Japan: a systematic literature review," The Open Rheumatology Journal, vol. 11, no. 1, pp. 43-52, 2017.

[3] A. D. Woolf and B. Pfleger, "Burden of major musculoskeletal conditions," Bulletin of World Health Organization, vol. 81, no. 9, pp. 646-656, 2003.

[4] Y. Miwa, R. Takahashi, Y. Ikari et al., "Clinical characteristics of rheumatoid arthritis patients achieving functional remission with six months of biological DMARDs treatment," Internal Medicine, vol. 56, no. 8, pp. 903-906, 2017.

[5] M. L. L. Prevoo, M. A. Van'T Hof, H. H. Kuper, M. A. van Leeuwen, L. B. A. van de Putte, and P. L. C. M. van Riel, "Modified disease activity scores that include twenty-eight-joint counts development and validation in a prospective longitudinal study of patients with rheumatoid arthritis," Arthritis \& Rheumatism, vol. 38, no. 1, pp. 44-48, 1995.

[6] T. C. Lee, J. Cui, B. Lu, M. L. Frits et al., "Pain persists in DAS28 rheumatoid arthritis remission but not in ACR/ EULAR remission: a longitudinal observational study," Arthritis Research \& Therapy, vol. 13, no. 3, p. R83, 2011.

[7] S. Ahmed, T. Magan, M. Vargas, A. Harrison, and N. Sofat, "Use of the pain detect tool in rheumatoid arthritis suggests neuropathic and sensitization components in pain reporting," Journal of Pain Research, vol. 7, pp. 579-588, 2014.

[8] M. Purabdollah, S. Lakdizaji, A. Rahmani, M. Hajalilu, and K. Ansarin, "Relationship between sleep disorders, pain and quality of life in patients with rheumatoid arthritis," Scandinavian Journal of Caring Sciences, vol. 4, no. 3, pp. 223-241, 2015.

[9] B. Börsbo, B. Gerdle, and M. Peolsson, "Impact of the interaction between self-efficacy, symptoms and catastrophising on disability, quality of life and health in with chronic pain patients," Disability and Rehabilitation, vol. 32, no. 17, pp. 1387-1396, 2010.

[10] J. A. Sturgeon and A. J. Zautra, "State and trait pain catastrophizing and emotional health in rheumatoid arthritis," Annals of Behavioral Medicine, vol. 45, no. 1, pp. 69-77, 2013.

[11] K. Izumi, Y. Kaneko, H. Yasuoka et al., "Tocilizumab is clinically, functionally, and radiographically effective and safe either with or without low-dose methotrexate in active rheumatoid arthritis patients with inadequate responses to DMARDs and/or TNF inhibitors: a single-center retrospective cohort study (KEIO-TCZ study) at week 52," Modern Rheumatology, vol. 25, no. 1, pp. 31-37, 2015.

[12] J. T. Joensuu, S. Huoponen, K. J. Aaltonen, Y. T. Konttinen, D. Nordström, and M. Blom, "The cost-effectiveness of biologics for the treatment of rheumatoid arthritis: a systematic review," PLoS One, vol. 10, no. 3, Article ID e0119683, 2015.

[13] A. N. Mian, F. Ibrahim, D. L. Scott, and J. Galloway, "Optimal responses in disease activity scores to treatment in rheumatoid arthritis: is a DAS28 reduction of $>1.2$ sufficient?" Arthritis Research \& Therapy, vol. 18, no. 1, p. 142, 2016. 
[14] P. Dolan, "Modeling valuations for EuroQol health states," Medical Care, vol. 35, no. 11, pp. 1095-1108, 1997.

[15] M. Herdman, C. Gudex, A. Lloyd et al., "Development and preliminary testing of the new five-level version of EQ-5D (EQ-5D-5L)," Quality of Life Research, vol. 20, no. 10, pp. 1727-1736, 2011.

[16] T. Shiroiwa, S. Ikeda, S. Noto et al., "Comparison of value set based on DCE and/or TTO data: scoring for EQ-5D-5L health states in Japan," Value in Health, vol. 19, no. 5, pp. 648-654, 2016.

[17] O. Efthymiadou, J. Mossman, and P. Kanavos, "Health related quality of life aspects not captured by EQ-5D-5L: results from an international survey of patients," Health Policy, vol. 123, no. 2, pp. 159-165, 2019.

[18] V. Tóthová, S. Bártlová, F. Dolák et al., "Quality of life in patients with chronic diseases," Neuroendocrinology Letters, vol. 35, no. 1, pp. 11-18, 2014.

[19] M. Hernández-Alava and S. Pudney, "Econometric modelling of multiple self-reports of health states: the switch from EQ5D-3L to EQ-5D-5L in evaluating drug therapies for rheumatoid arthritis," Journal of Health Economics, vol. 55, pp. 139-152, 2017.

[20] D. Aletaha, T. Neogi, A. J. Silman et al., "2010 rheumatoid arthritis classification criteria: an American College of Rheumatology/European League against Rheumatism collaborative initiative," Annals of Rheumatic Diseases, vol. 69, no. 9, pp. 1580-1588, 2010.

[21] G. Wells, J.-C. Becker, J. Teng et al., "Validation of the 28-joint Disease Activity Score (DAS28) and European league against Rheumatism response criteria based on C-reactive protein against disease progression in patients with rheumatoid arthritis, and comparison with the DAS28 based on erythrocyte sedimentation rate," Annals of the Rheumatic Diseases, vol. 68, no. 6, pp. 954-960, 2009.

[22] E. Inoue, H. Yamanaka, M. Hara, T. Tomatsu, and N. Kamatani, "Comparison of disease activity score (DAS)28erythrocyte sedimentation rate and DAS28- C-reactive protein threshold values," Annals of the Rheumatic Diseases, vol. 66, no. 3, pp. 407-409, 2007.

[23] R. Freynhagen, R. Baron, U. Gockel, and T. R. Tölle, "painDETECT: a new screening questionnaire to identify neuropathic components in patients with back pain," Current Medical Research and Opinion, vol. 22, no. 10, pp. 1911-1920, 2006.

[24] M. K. Nicholas, "The pain self-efficacy questionnaire: taking pain into account," European Journal of Pain, vol. 11, no. 2, pp. 153-163, 2007.

[25] T. Adachi, A. Nakae, T. Maruo et al., "Validation of the Japanese version of the pain self-efficacy questionnaire in Japanese patients with chronic pain," Pain Medicine, vol. 15, no. 8, pp. 1405-1417, 2014.

[26] M. J. L. Sullivan, S. R. Bishop, and J. Pivik, "The pain catastrophizing scale: development and validation," Psychological Assessment, vol. 7, no. 4, pp. 524-532, 1995.

[27] H. Matsuoka and Y. Sakano, "Assessment of cognitive aspect of pain: development, reliability, and validation of Japanese version of pain catastrophizing scale," Japanese Journal of Psychosomatic Medicine, vol. 47, no. 2, pp. 95-102, 2007.

[28] L. Li, S. L. Bokshan, S. R. Mehta, and B. D. Owens, "Disparities in cost and access by caseload for arthroscopic rotator cuff repair: an analysis of 18,616 cases," Orthopaedic Journal of Sports Medicine, vol. 7, no. 6, 2019.

[29] K. K. Hall and P. R. Scheuerman, "Development of multiple regression models to predict sources of fecal pollution," Water Environment Research, vol. 89, no. 11, pp. 1961-1969, 2017.
[30] D. A. Rubin, "MRI and ultrasound of the hands and wrists in rheumatoid arthritis. I. imaging findings," Skeletal Radiology, vol. 48, no. 5, pp. 677-695, 2019.

[31] J. K. Anderson, L. Zimmerman, L. Caplan, and K. Michaud, "Measures of rheumatoid arthritis disease activity: patient (PtGA) and provider (PrGA) global assessment of disease activity, disease activity score (DAS) and disease activity score with 28-joint counts (DAS28), simplified disease activity index (SDAI), clinical disease activity index (CDAI), patient Activity score (PAS) and patient Activity score-II (PASII), routine assessment of patient index data (RAPID), rheumatoid arthritis disease activity index (RADAI) and rheumatoid arthritis disease activity index-5 (RADAI-5), chronic arthritis systemic index (CASI), patient-based disease activity score with ESR (PDAS1) and patient-based disease activity score without ESR (PDAS2), and mean overall index for rheumatoid arthritis (MOI-RA)," Arthritis Care \& Research (Hoboken), vol. 63, no. Suppl. 11, pp. S14-S36, 2011.

[32] K. Romanowska-Próchnicka, M. Olesińska, A. ParadowskaGorycka et al., "Discrepancies in assessment of patients with rheumatoid arthritis and secondary Sjögren's syndrome by DAS28-ESR and DAS28-CRP," Central European Journal of Immunology, vol. 2, no. 2, pp. 188-194, 2016.

[33] W. Hamdi, O. Néji, M. M. Ghannouchi, D. Kaffel, and M. M. Kchir, "Comparative study of indices of activity evaluation in rheumatoid arthritis," Annals of Physical and Rehabilitation Medicine, vol. 54, no. 7, pp. 421-428, 2011.

[34] P. M. J. Welsing, R. B. M. Landewé, P. L. C. M. van Riel et al., "The relationship between disease activity and radiologic progression in patients with rheumatoid arthritis: a longitudinal analysis," Arthritis \& Rheumatism, vol. 50, no. 7, pp. 2082-2093, 2004.

[35] K. M. Son, S. H. Song, S. K. Lim, Y. I. Seo, and H. A. Kim, "Characteristics of patients with rheumatoid arthritis in clinical remission: the many aspects of DAS28 remission," Clinical and Experimental Rheumatology, vol. 30, no. 6, pp. 947-950, 2012.

[36] M. Mierau, M. Schoels, G. Gonda, J. Fuchs, D. Aletaha, and J. S. Smolen, "Assessing remission in clinical practice," Rheumatology, vol. 46, no. 6, pp. 975-979, 2007.

[37] P. Sewerin, S. Vordenbaeumen, A. Hoyer et al., "Silent progression in patients with rheumatoid arthritis: is DAS28 remission an insufficient goal in RA? Results from the German Remission-plus cohort," BMC Musculoskeletal Disorders, vol. 18, no. 1, p. 163, 2017.

[38] S. Rifbjerg-Madsen, A. W. Christensen, M. Boesen et al., "Can the painDETECT questionnaire score and MRI help predict treatment outcome in rheumatoid arthritis: protocol for the Frederiksberg hospital's Rheumatoid Arthritis, pain assessment and Medical Evaluation (FRAME-cohort) study," BMJ Open, vol. 4, no. 11, Article ID e006058, 2014.

[39] S. M. Koop, P. M. ten Klooster, H. E. Vonkeman, L. M. Steunebrink, and M. A. van de Laar, "Neuropathic-like pain features and cross-sectional associations in rheumatoid arthritis," Arthritis Research \& Therapy, vol. 17, p. 237, 2015.

[40] A. Christensen, S. Rifbjerg-Madsen, R. Christensen et al., "Non-nociceptive pain in rheumatoid arthritis is frequent and affects disease activity estimation: cross-sectional data from the FRAME study," Scandinavian Journal of Rheumatology, vol. 45, no. 6, pp. 461-469, 2016.

[41] A. Bandura, Social Foundations of Thought and Action: A Social Cognitive Theory, Prentice-Hall, Englewood Cliffs, NJ, USA, 1986.

[42] L. Ackermans, M. G. Hageman, A. H. Bos, D. Haverkamp, V. A. B. Scholtes, and R. W. Poolman, "Feedback to patients 
about patient-reported outcomes does not improve empowerment or satisfaction," Clinical Orthopaedics and Related Research, vol. 476, no. 4, pp. 716-722, 2018.

[43] R. Blamey, K. Jolly, S. Greenfield, and P. Jobanputra, "Patterns of analgesic use, pain and self-efficacy: a cross-sectional study of patients attending a hospital rheumatology clinic," $B M C$ Musculoskeletal Disorders, vol. 10, p. 137, 2009.

[44] P. Campbell, N. E. Foster, E. Thomas, and K. M. Dunn, "Prognostic indicators of low back pain in primary care: fiveyear prospective study," The Journal of Pain, vol. 14, no. 8, pp. 873-883, 2013.

[45] R. Chester, C. Jerosch-Herold, J. Lewis, and L. Shepstone, "Psychological factors are associated with the outcome of physiotherapy for people with shoulder pain: a multicentre longitudinal cohort study," British Journal of Sports Medicine, vol. 52, no. 4, pp. 269-275, 2018.

[46] I. Ahlstrand, S. Vaz, T. Falkmer, I. Thyberg, and M. Björk, "Self-efficacy and pain acceptance as mediators of the relationship between pain and performance of valued life activities in women and men with rheumatoid arthritis," Clinical Rehabilitation, vol. 31, no. 6, pp. 824-834, 2017.

[47] P. J. Quartana, C. M. Campbell, and R. R. Edwards, "Pain catastrophizing: a critical review," Expert Review of Neurotherapeutics, vol. 9, no. 5, pp. 745-758, 2009.

[48] S. Cho, H.-Y. Kim, and J.-H. Lee, "Validation of the Korean version of the Pain Catastrophizing Scale in patients with chronic non-cancer pain," Quality of Life Research, vol. 22, no. 7, pp. 1767-1772, 2013.

[49] M. Penhoat, A. Saraux, B. Le Goff, P. Augereau, Y. Maugars, and J.-M. Berthelot, "High pain catastrophizing scores in onefourth of patients on biotherapy for spondylarthritis or rheumatoid arthritis," Joint Bone Spine, vol. 81, no. 3, pp. 235-239, 2014.

[50] S. C. Hayes, "Acceptance and commitment therapy, relational frame theory, and the third wave of behavioral and cognitive therapies-republished article," Behavior Therapy, vol. 47, no. 6, pp. 869-885, 2016.

[51] L. S. Hughes, J. Clark, J. A. Colclough, E. Dale, and D. McMillan, "Acceptance and commitment therapy (ACT) for chronic pain," The Clinical Journal of Pain, vol. 33, no. 6, pp. 552-568, 2017.

[52] R. K. Wicksell, G. L. Olsson, and S. C. Hayes, "Mediators of change in acceptance and commitment therapy for pediatric chronic pain," Pain, vol. 152, no. 12, pp. 2792-2801, 2011.

[53] R. J. E. M. Smeets, J. W. S. Vlaeyen, A. D. M. Kester, and J. A. Knottnerus, "Reduction of pain catastrophizing mediates the outcome of both physical and cognitive-behavioral treatment in chronic low back pain," The Journal of Pain, vol. 7, no. 4, pp. 261-271, 2006.

[54] A. Hashimoto, H. Sato, Y. Nishibayahi et al., "A multicenter cross-sectional study on the health related quality of life of patients with rheumatoid arthritis using a revised Japanese version of the arthritis impact measurement scales version 2 (AIMS 2), focusing on physical disability and its associative factors," Ryumachi, vol. 41, no. 1, pp. 9-24, 2001, in Japanese.

[55] M. Boers, A. C. Verhoeven, H. M. Markusse et al., "Randomised comparison of combined step-down prednisolone, methotrexate and sulphasalazine with sulphasalazine alone in early rheumatoid arthritis," Lancet, vol. 350, no. 9074, pp. 309-318, 1997.

[56] M. Lapčević, M. Vuković, B. S. Gvozdenović, V. Mioljević, and S. Marjanović, "Socioeconomic and therapy factor influence on self-reported fatigue, anxiety and depression in rheumatoid arthritis patients," Revista Brasileira de Reumatologia, vol. 57, no. 6, pp. 545-556, 2017.

[57] J. M. Davis 3rd. and E. L. Matteson, "My treatment approach to rheumatoid arthritis," Mayo Clinic Proceedings, vol. 87, no. 7, pp. 659-673, 2012.

[58] Y. M. Chiu, M. S. Lai, H. Y. Lin, H. C. Lang, L. J. Lee, and J. D. Wang, "Disease activity affects all domains of quality of life in patients with rheumatoid arthritis and is modified by disease duration," Clinical and Experimental Rheumatology, vol. 32, no. 6, pp. 898-903, 2014.

[59] I. Wysocka-Skurska, M. Sierakowska, and W. Kułak, "Evaluation of quality of life in chronic, progressing rheumatic diseases based on the example of osteoarthritis and rheumatoid arthritis," Clinical Interventions in Aging, vol. 11, pp. 1741-1750, 2016.

[60] B. Kwiatkowska, A. Kłak, M. Maślińska, M. Mańczak, and F. Raciborski, "Factors of depression among patients with rheumatoid arthritis," Reumatologia/Rheumatology, vol. 56, no. 4 , pp. 219-227, 2018.

[61] S. G. Hofmann, J. Curtiss, J. K. Carpenter, and S. Kind, "Effect of treatments for depression on quality of life: a meta-analysis," Cognitive Behavioral Therapy, vol. 46, no. 4, pp. 264286, 2017.

[62] S. Wattanasoei, B. Binson, R. Kumar, R. Somrongthong, and N. Kanchanakhan, "Quality of life through listening music among elderly people in semi-urban area, Thailand," Journal of Ayub Medical College Abbottabad, vol. 29, no. 1, pp. 21-25, 2017.

[63] H. El Tumi, M. I. Johnson, P. B. F. Dantas, M. J. Maynard, and O. A. Tashani, "Age-related changes in pain sensitivity in healthy humans: a systematic review with meta-analysis," European Journal of Pain, vol. 21, no. 6, pp. 955-964, 2017.

[64] J. S Smolen, F. C. Breedveld, G. R. Burmester et al., "Treating rheumatoid arthritis to target: 2014 update of the recommendations of an international task force," Annals of Rheumatic Diseases, vol. 75, no. 1, pp. 3-15, 2016. 